\title{
Encaminhamentos aos cuidados paliativos para pacientes com câncer: revisão integrativa
}

\author{
Referrals to palliative care for patients with cancer: an \\ integrative review
}

\author{
Lailah Maria Pinto Nunes ${ }^{1}$ - Zenith Rosa Silvino² • Marlea Chagas Moreira ${ }^{3} \bullet$ Taiza Florencio Costa ${ }^{\bullet}$ \\ Barbara Pompeu Christovam ${ }^{5}$
}

\section{RESUMO}

No cenário oncológico atual temos o surgimento de novas drogas e tecnologias para prolongamento de vida. Com isso tivemos a promessa de aumento de sobrevida aliada a dilemas éticos como qual o momento ideal para encaminhamento aos cuidados paliativos. Objetivo: Analisar as evidências disponíveis na literatura acerca de consultas e encaminhamentos aos Cuidados Paliativos para pacientes com câncer. Método: Revisão integrativa nas bases de dados LILACS e Medline. Utilizados os descritores e palavras-chave: "consultation and referral","palliative care", "cancer", "encaminhamento e consulta" e "câncer" acompanhados do conector "and". Resultados: Artigos alocados em duas categorias: 1. Artigos sobre Momento dos Encaminhamentos aos Cuidados Paliativos e 2. Artigos sobre Características dos encaminhados aos Cuidados Paliativos. Artigos sobre momento do encaminhamento abordaram encaminhamentos precoce, tardios e encaminhamentos ao hospice. Artigos sobre características dos encaminhamentos abordaram aspectos facilitadores e barreiras aos encaminhamentos. Conclusões: Apesar dos benefícios apontados pelos encaminhamentos precoces ainda vemos na prática o encaminhamento tardio aos cuidados paliativos. Esforços para conscientização da equipe de saúde e aumento da oferta de serviços de cuidados paliativos parecem boas estratégias para reverter esta realidade.

Palavras-chave: Referência e consulta; Cuidados paliativos; Neoplasias.

\begin{abstract}
In the current oncology scenario we face the dicovery of new drugs and technologies for prolonging life. Thus we have the promise of increased survival, ethical dilemmas such as the ideal time for referral to palliative care have emerged. Aim: To review the evidence available in literature about consultations and referrals to palliative care for cancer patients. Method: Integrative review in LILACS and Medline databases. The search strategie included the use of the terms: "consultation and referral," "palliative care," "cancer," "referral and consultation" and "cancer" accompanied by "and". Results: Articles were allocated into two categories: 1 . Articles about moment of referrals to Palliative care and 2. Articles about Characteristics of referral to palliative care. Articles on the moment of referrals addressed early, late and hospice referrals. Articles on characteristics of referrals addressed facilitating factors and barriers to referrals. Conclusions: Despite the benefits mentioned we still see in practice late referrals to palliative care. Efforts to raise awareness of the health team and increasing the supply of palliative care services seems as good strategies to reverse this reality.
\end{abstract}

Keywords: Referral and consultation; Palliative care; Neoplasms.

${ }_{1}^{1}$ Discente do MPEA/UFF. Especialista em Oncologia pelo INCA. Enfermeira da Central de Quimioterapia do HCIII/INCA, Rio de Janeiro (RJ), Brasil. E-mail: lailahnunes@yahoo.com.br.

${ }^{2}$ Doutora em Enfermagem. Professora-Titular da Escola de Enfermagem Aurora de Afonso Costa da UFF, Niterói (RJ), Brasil. E-mail: zenithrosa@terra.com.br.

${ }_{3}^{3}$ Doutora em Enfermagem. Professora Associada da Universidade Federal do Rio de Janeiro (RJ), Brasil. E-mail: marleachagas@gmail.com.br

${ }^{4}$ Doutora em Enfermagem. Professor Adjunto da Escola de Enfermagem Aurora de Afonso Costa da UFF (RJ), Brasil. E-mail: taizaflorencio@hotmail.com

${ }^{5}$ Doutorado em Enfermagem. Diretora do CAIT Mazzine Bueno da UFF (RJ), Brasil. E-mail: babypompeu@gmail.com. 


\section{INTRODUÇÃO}

As mudanças ocorridas com 0 envelhecimento populacional, mudanças sanitárias e nas relações entre as pessoas e o ambiente ocasionaram uma alteração no perfil de morbimortalidade brasileiro levando a diminuição na ocorrência das doenças infectocontagiosas e colocando as doenças crônico-degenerativas como centro de atenção dos problemas de doença e morte da população. Dentre essas doenças se destaca o câncer cuja estimativa para o ano de 2014, que será válida também para o ano de 2015, aponta para a ocorrência de aproximadamente 576 mil casos novos $^{1}$.

Apesar de todos os esforços empregados na detecção precoce, alguns tipos de câncer, como o de mama e pulmão, apresentam altas taxas de mortalidade atribuídas ao diagnostico tardio ${ }^{1}$. Como reflexo desta situação temos uma grande demanda de pacientes com doença avançada, que não mais se beneficiarão de tratamentos curativos ${ }^{2}$.

Os pacientes com doença metastática são considerados fora de possibilidade terapêutica de cura, porém poderão se beneficiar de tratamentos para prolongamento de sobrevida e melhoria da qualidade de vida conhecidos como tratamentos paliativos ${ }^{3-4}$.

Nas últimas décadas vivencia-se no cenário oncológico - surgimento de novas drogas e tecnologias para prolongamento de vida. Se por um lado houve a promessa de aumento de sobrevida, nos deparamos hoje com dilemas éticos como qual o momento ideal para interrupção de medidas terapêuticas em pacientes sem possibilidades de cura e introdução das medidas de suporte exclusivo, conhecidas como cuidados paliativos².

A partir de 2002, houve uma mudança na definição de Cuidados Paliativos (CP) pela Organização Mundial de Saúde $(\mathrm{OMS})^{5}$ que passou a adotar os conceitos de abordagem precoce, cuidados multidisciplinares e o foco na melhoria na qualidade de vida. Esta definição trouxe uma visão mais ampla dos $C P$, para além dos cuidados prestados em fim de vida ${ }^{6}$.

Nesse sentido, cada vez mais estão sendo estudados modelos de cuidado integrado associando os CP com o tratamento para o câncer. $\mathrm{O}$ objetivo do cuidado integrado é ampliar a oferta de CP, a partir da detecção de câncer incurável de modo a suprir às necessidades de pacientes de uma forma mais global'.

Com isso, o objetivo desse estudo é analisar as evidências disponíveis na literatura acerca de consultas e encaminhamentos aos Cuidados Paliativos para pacientes com câncer.

\section{MÉTODO}

alavras-chaveA prática baseada em evidências é a construção do processo de tomada de decisão a partir do uso racional das evidências científicas sobre determinado tema/assunto ${ }^{8}$. Sua relevância para a enfermagem está no seu potencial de atuação na gestão do cuidado, guiando o estabelecimento de prioridades, o dimensionamento do tempo do cuidado e proporcionado uma assistência de enfermagem mais eficaz e satisfatória ${ }^{8-9}$.

Para toda boa construção precisa-se de um alicerce sólido, confiável. Por isso, precisamos de uma seleção criteriosa e adequada da literatura científica disponível. Assim, como nossa temática está voltada para uma questão ampla, que envolve os encaminhamentos e consultas de CP, optou-se pela ferramenta da revisão integrativa, definida como técnica sistematizada de busca de literatura que permite a síntese de múltiplos estudos publicados e possibilita conclusões gerais a respeito de uma particular área de estudo ${ }^{10}$.

Esta revisão foi realizada em seis etapas a saber: 1) elaboração da questão norteadora seguida pela busca dos descritores; 2) seleção da mostra através de critérios de inclusão e exclusão; 3) caracterização dos estudos: 4) análise crítica dos estudos; 5) discussão e interpretação dos resultados; 6) apresentação da revisão e síntese do conhecimento ${ }^{10}$.

Para elaboração da questão norteadora utilizamos a estratégia PICO (Acrônimo formado pelas primeiras letras das palavras em inglês Paciente, Intervenção, Comparação e Desfecho) ${ }^{8}$. 0 ponto de partida foi a questão de pesquisa: Quais as evidências disponíveis na literatura acerca de encaminhamentos e consultas em cuidados paliativos para pacientes com câncer?

O levantamento dos dados ocorreu durante o período de Setembro a Outubro de 2015, utilizando estudos publicados em Janeiro de 2010 a Outubro de 2015, a partir das bases de dados LILACS - Literatura Latino-Americana e do Caribe em Ciências da Saúde e MEDLINE - Medical Literature Analysis and Retrieval System Online.

Como critérios de inclusão foram usados: artigos publicados nos últimos 5 anos, em humanos adultos, publicados em inglês, português e espanhol. Como critérios de exclusão foram estipulados: Relatos de caso e artigos de opinião, artigos em cuidados paliativos não exclusivamente a pacientes com câncer, artigos sobre familiares de pacientes com câncer, terapias paliativas que não relacionadas aos encaminhamentos (ex. consultas cirúrgicas, radioterápicas, intervenções para dor, estudos clínicos).

Foram utilizados os descritores e palavras-chave, selecionados a partir da estratégia PICO:"cancer" (paciente), "consultation and referral"(intervenção), "palliative care"(desfecho), do MeSH (Banco de descritores de artigos indexados no MEDLINE) associados ao conector AND para cruzamento dos termos.

Para complementar a pesquisa e obedecendo aos mesmos critérios de inclusão e exclusão, utilizou-se os descritores e palavras chave: "referência e consulta" e "cancer" da Biblioteca Virtual em Saúde (BVS) para também associado ao concector AND.

As etapas da revisão forma realizadas da seguinte forma: 


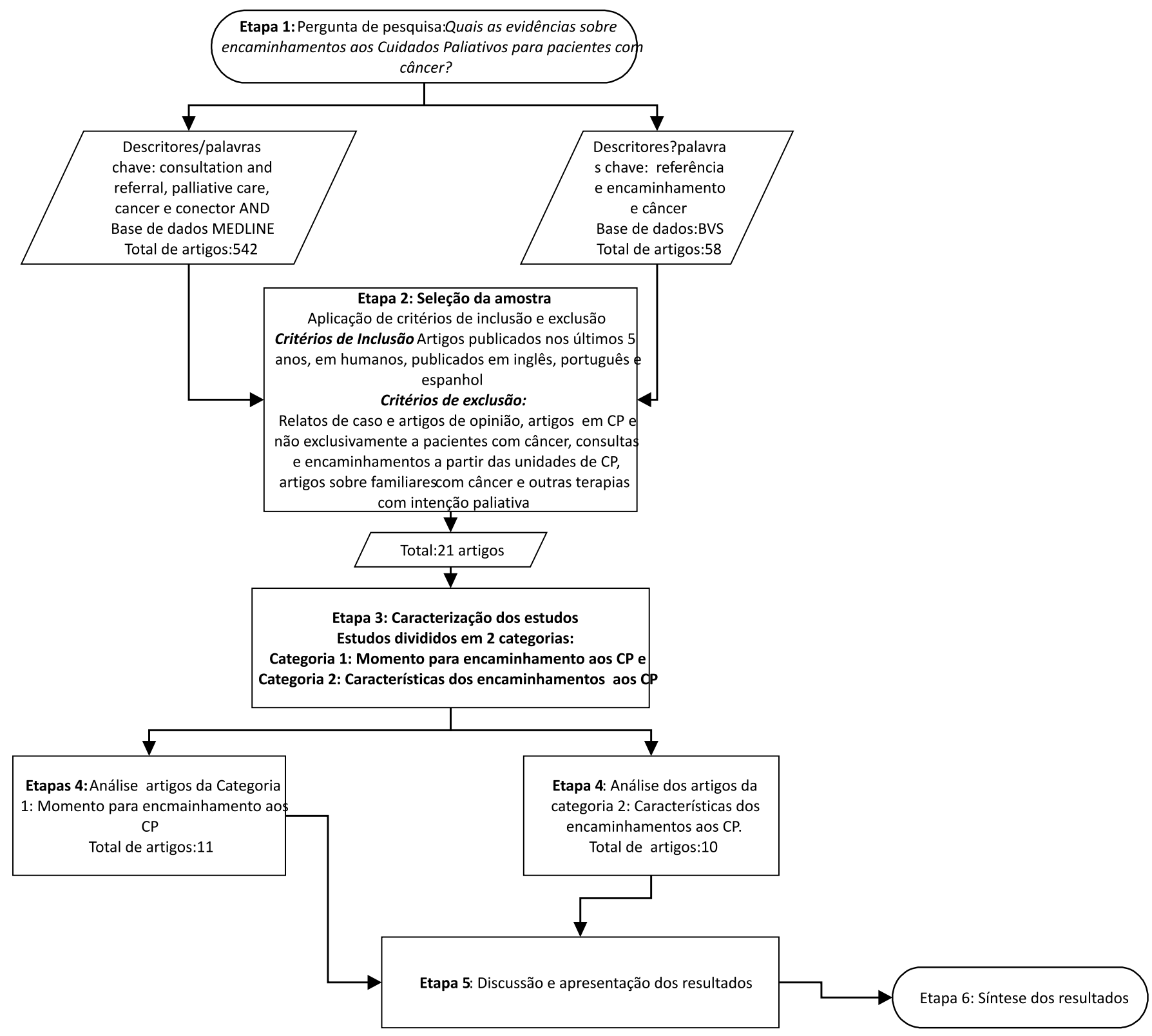

Figura 1: Fluxograma das etapas da revisão integrativa sobre encaminhamentos em cuidados paliativos para pacientes com câncer. Rio de Janeiro, 2015.

\section{RESULTADOS E DISCUSSÃO}

Após a aplicação de critérios de exclusão e inclusão obtivemos um total de 21 artigos divididos em 2 categorias de análise: Categoria 1. Artigos sobre Momento dos Encaminhamentos aos Cuidados Paliativos e Categoria 2. Artigos sobre Características dos encaminhamentos aos Cuidados Paliativos.

\section{Categoria 1: momento para encaminhamento aos CP}

Nesta categoria foram abordados aspectos relacionados aos encaminhamentos precoces e tardios aos cuidados paliativos, além da análise do percentual de pacientes encaminhados com relação a localização do tumor primário. Foram encontrados 11 artigos sobre os seguintes temas:

Nesta categoria houve predomínio de artigos norte americanos. Neste país o encaminhamento para o hospice (unidade de internação em CP) é coberto pelo sistema de saúde (Medicare) por reembolso e requer para sua inclusão pacientes com expectativa de vida inferior a 6 meses e abdicação de qualquer terapia ativa para o câncer (como quimio ou radioterapia) ${ }^{22}$.

Esse sistema de saúde que opera por reembolso fomenta os encaminhamentos tardios aos CP, uma vez que obriga médicos e pacientes a escolherem entre medidas curativas e tratamento paliativo. Além disso, ao fornecerem cobertura a medidas de prolongamento de sobrevida e não reembolsarem de forma diferenciada consultas que envolvam os processos relacionados ao encaminhamento aos CP (discussões de metas de cuidado realistas com pacientes e familiares), acabam por favorecer a oferta de tratamentos agressivos e fúteis na fase de fim de vida ${ }^{22}$.

Como medida para diminuir esta distorção as entidades de classe americanas como a American Society of Clinical Oncology (ASCO) produziram uma declaração de política 
Quadro 1- Síntese dos artigos sobre o momento para encaminhamento aos CP

\begin{tabular}{|c|c|}
\hline Título/Ano & Objetivo \\
\hline $\begin{array}{l}\text { Emergency department-triggered palliative care in advanced } \\
\text { cancer: proof of concept/2015 }\end{array}$ & $\begin{array}{l}\text { Avaliar o processo de encaminhamento precoce a partir do } \\
\text { departamento de emergência para o serviço de CP de pacientes } \\
\text { com câncer avançado e incurável para comparar a proporção e } \\
\text { momento apropriado do momento de encaminhamento. }\end{array}$ \\
\hline $\begin{array}{l}\text { Early integration of palliative care facilitates the } \\
\text { discontinuation of anticancer treatment in women with } \\
\text { advanced breast or gynecologic cancers } / 2014^{12}\end{array}$ & $\begin{array}{l}\text { Avaliar indicadores de saúde em mulheres com câncer } \\
\text { ginecológico avançado após descontinuação do tratamento ativo } \\
\text { com relação ao modelo de cuidado recebido (encaminhamento } \\
\text { precoce X tratamento padrão). }\end{array}$ \\
\hline $\begin{array}{l}\text { Impact of timing and setting of palliative care referral on } \\
\text { quality of end-of-life care in cancer patients } / 2015^{13}\end{array}$ & $\begin{array}{l}\text { Examinar o momento de encaminhamento aos CP e sua relação } \\
\text { com indicadores de cuidado no fim de vida }\end{array}$ \\
\hline $\begin{array}{l}\text { Palliative care and hematological malignancies: increased } \\
\text { referrals at a comprehensive cancer center } / 2013^{14}\end{array}$ & $\begin{array}{l}\text { Analisar tendência em número e proporção dos } \\
\text { encaminhamentos aos serviços de CP e mudanças nas } \\
\text { características dos encaminhamentos. }\end{array}$ \\
\hline $\begin{array}{l}\text { Early palliative intervention for patients with advanced } \\
\text { cancer/2013 }\end{array}$ & $\begin{array}{l}\text { Comparação entre encaminhamentos tardios e precoces aos CP } \\
\text { para pacientes com câncer avançado e estabelecer momento } \\
\text { adequado para encaminhamento. }\end{array}$ \\
\hline $\begin{array}{l}\text { Early referral to supportive care specialists for symptom } \\
\text { burden in lung cancer patients: a comparison of non- } \\
\text { Hispanic whites, Hispanics, and non-Hispanic blacks } / 2012^{16}\end{array}$ & $\begin{array}{l}\text { Avaliar se o momento apropriado para encaminhamento ao CP } \\
\text { e resultados do encaminhamento na carga de sintomas teve } \\
\text { relação com raça e etnia em pacientes com câncer em hospital } \\
\text { terciário. }\end{array}$ \\
\hline $\begin{array}{l}\text { Hospice referrals and code status: outcomes of inpatient } \\
\text { pallaitve care consultation among asian americasn and } \\
\text { pacific islanders with cancer/2011 } 17\end{array}$ & $\begin{array}{l}\text { Comparar encaminhamentos de pacientes com câncer ao hospice } \\
\text { com relação a critérios como mudança de status funcional após } \\
\text { consulta, etnia e planejamento de cuidados. }\end{array}$ \\
\hline $\begin{array}{l}\text { Components of early outpatient palliative care consultation } \\
\text { in patients with metastatic nonsmall cell lung cancer/2011 }\end{array}$ & $\begin{array}{l}\text { Descrever prática de encaminhamento precoce aos CP e melhora } \\
\text { na qualidade de vida, humor e sobrevida de pacientes com } \\
\text { câncer. }\end{array}$ \\
\hline $\begin{array}{l}\text { Late referrals to home palliative care service affecting death } \\
\text { at home in advanced cancer patients in Japan: a nationwide } \\
\text { survey/2011 }\end{array}$ & $\begin{array}{l}\text { Identificar fatores que influenciaram o local da de morte entre } \\
\text { pacientes recebendo CP domiciliar, com foco na identificação do } \\
\text { momento apropriado ao encaminhamento ao home care. }\end{array}$ \\
\hline Late referral to palliative care services in Korea/201120 & Investigar padrões de encaminhamento precoce e tardios. \\
\hline $\begin{array}{l}\text { Association between a name change from palliative to } \\
\text { supportive care and the timing of patient referrals at a } \\
\text { comprehensive cancer center/2011 }\end{array}$ & $\begin{array}{l}\text { Comparar características e disposição para alta de pacientes } \\
\text { hospitalizados com câncer de pulmão com relação ao } \\
\text { encaminhamento para CP X tratamento padrão. }\end{array}$ \\
\hline
\end{tabular}

em 2011 intitulada American Society of Clinical Oncology Statement: Toward Individualized Care for Patients With Advanced Cancer que aponta o modelo de CP concomitante a terapia voltada para o câncer avançado como uma das estratégias para vencer as barreiras de encaminhamento destes pacientes ao serviço de hospice, inclusive apontando que programas que usam esta estratégia devem ser ampliados e apoiados ${ }^{7}$. Essa recomendação é endossada pela declaração da Oncology Nursing Society em 2014.

Os benefícios do encaminhamento precoce foram descritos em artigo publicado em $2010^{23}$ e servem de referência para os artigos de encaminhamento precoce devido a relevância de seus achados, principalmente a melhora na sobrevida de pacientes com câncer de pulmão que receberam consultas antecipadas de CP, equiparando o efeito dessas consultas ao uso da quimioterapia no prolongamento da sobrevida ${ }^{23}$.

Quanto a população priorizada nos artigos para encaminhamento precoce foram apontados pacientes com tumores ginecológicos, de pulmão e de cabeça e pescoço. Estas patologias compartilham prognóstico ruim (expectativa de vida < 12 meses) e alta carga de sintomas requerendo constantes visitas aos serviços de emergência, internações, tornando essas populações interessantes sobre o ponto de vista de estudos intervencionistas, de uso de serviços ou sobrevida ${ }^{24-27}$. Neste tipo de estudo é importante a seleção de uma amostra homogênea e representativa para atingir significado estatístico. Sendo assim, é correto classificar os pacientes com relação a uma medida de prognóstico (expectativa de sobrevida).

Pacientes hematológicos apresentam características que os tornam uma população com maiores índices de encaminhamentos tardios. O curso da doença hematológica varia bastante, desde pacientes com linfoma que podem sobreviver longos períodos de tempo sem requerer tratamento quimioterápico até pacientes com leucemia aguda que necessitam de regimes intensos de tratamento ${ }^{28}$.

Os índices de cura para tumores hematológicos aumentam quando uma terapia mais agressiva é empregada, porém também aumentam os riscos de complicações que ameaçam a vida, como sepse e sangramentos. Por conta 
deste limite tênue entre terapia curativa e declínio clínico, um grande número de pacientes hematológicos podem ter seu risco para morte e complicações dos tratamentos subestimados, e consequentemente, são mais tardiamente encaminhados aos serviços de $\mathrm{CP}^{28}$.

Deummodogeralforamconsideradosencaminhamentos precoces aos CP desde encaminhamentos a partir da emergência, encaminhamentos de pacientes com expectativa de vida de até 2 anos, pacientes recebendo tratamento ativo para doença, pacientes recebendo tratamento ambulatorial desde 7 dias até 3 meses antes da morte.

Encaminhamentos precoces foram associados a diversos benefícios como: antecipação do recebimento da consulta de CP, menores internações na emergência, hospitalizações e mortes na emergência nos últimos 30 dias de vida, aumento na sobrevida, menos depressão, maior qualidade de vida e sobrevida. Além disso, foram associados a prestação de $\mathrm{CP}$ ambulatorial e à maiores índices de tratamento agressivo, pois permitiram que pacientes pudessem suportar o tratamento por mais tempo.

Os pacientes encaminhados precocemente foram relacionados às seguintes patologias: câncer de pulmão não pequenas células, coloretal e tumores de cabeça e pescoço. Já encaminhamentos considerados tardios foram relacionados a ocorrência de tumores hematológicos e pacientes do sexo masculino.
Encaminhamentos tardios variaram igualmente em definição com relação ao momento para encaminhamento. Estão presentes mesmo em serviços que provem $\mathrm{CP}$ ambulatorial e em patologias com prognóstico sabidamente reservado (ex. tumores cerebrais). Também estiveram associados a fatores socioeconômicos como tipo e modalidade de seguro saúde.

No Brasil carecemos de uma política nacional em Cuidados Paliativos, estando estes inseridos nas políticas oncológicas nos diversos níveis de atenção ${ }^{29-31}$. Como consequência, não existem indicadores para medir a execução e os encaminhamentos aos $\mathrm{CP}$, potencialmente contribuindo para aumentar a distorção a favor do tratamento oncológico padrão.

\section{Categoria 2: características do encaminhamento aos CP}

Nesta categoria foram abordados aspectos facilitadores e barreiras aos encaminhamentos aos CP. Foram encontrados 10 artigos com as seguintes temáticas:

Serviram de gatilho para os encaminhamentos aos $\mathrm{CP}$ a presença de declínio funcional e sintomas de difícil controle pelos pacientes. As características de declínio físico e funcional foram descritos através da presença de determinados sintomas (ex: déficit cognitivo e comportamental em pacientes com glioma ou obstrução

Quadro 2- Síntese dos artigos sobre características do encaminhamento ao CP

\begin{tabular}{|c|c|}
\hline Título/Ano & Objetivo \\
\hline $\begin{array}{l}\text { Hospice admissions for cancer in the final days of life: } \\
\text { independent predictors and implications for quality } \\
\text { measures } / 2014^{32}\end{array}$ & $\begin{array}{l}\text { Determinar características de pacientes encaminhadas ao } \\
\text { hospice nos últimos } 3 \text { dias de vida e descrever proporções de } \\
\text { encaminhamentos tardios. }\end{array}$ \\
\hline $\begin{array}{l}\text { Clinical presentation and patterns of care for short-term } \\
\text { survivors of malignant glioma/ } 2014^{33}\end{array}$ & $\begin{array}{l}\text { Descrever a apresentação clínica de pacientes com glioma } \\
\text { em período de fim de vida (<120 dias) e indicadores de CP e } \\
\text { cuidados de fim de vida. }\end{array}$ \\
\hline $\begin{array}{l}\text { Predictors of palliative care consultation on an inpatient } \\
\text { gynecologic oncology service: are we following ASCO } \\
\text { recommendations?/2014 }\end{array}$ & $\begin{array}{l}\text { Determinar preditores de consulta paliativa a pacientes } \\
\text { internados e caracterizar encaminhamentos com relação às } \\
\text { recomendações da Sociedade americana de Oncologia Clínica. }\end{array}$ \\
\hline $\begin{array}{l}\text { Oncologist factors that influence referrals to subspecialty } \\
\text { palliative care clinics } / 2014^{35}\end{array}$ & $\begin{array}{l}\text { Fatores que influenciam o encaminhamento ambulatorial aos } \\
\text { serviços de CP. }\end{array}$ \\
\hline $\begin{array}{l}\text { Clinical characteristics of cancer patients referred early to } \\
\text { supportive and palliative care } / 2013^{36}\end{array}$ & $\begin{array}{l}\text { Comparar características clínicas entre pacientes encaminhados } \\
\text { precoce e tardiamente aos CP }\end{array}$ \\
\hline $\begin{array}{l}\text { What influences the willingness of community physicians } \\
\text { to provide palliative care for patients with terminal cancer? } \\
\text { Evidence from a nationwide survey/ } 2013^{37}\end{array}$ & $\begin{array}{l}\text { Identificar a intenção de encaminhamento de médicos } \\
\text { comunitários aos serviços e CP e fatores que influenciam essa } \\
\text { intenção. }\end{array}$ \\
\hline $\begin{array}{l}\text { Access to palliative care among patients treated at a } \\
\text { comprehensive cancer center/2012 }\end{array}$ & $\begin{array}{l}\text { Determinar proporção de pacientes com câncer que receberam } \\
\text { CP e preditores do encaminhamento. }\end{array}$ \\
\hline $\begin{array}{l}\text { Referral practices of oncologists to specialized palliative } \\
\text { care/2012 }\end{array}$ & Descrever práticas de encaminhamento aos serviços de CP. \\
\hline $\begin{array}{l}\text { Lung cancer physicians' referral practices for palliative care } \\
\text { consultation } / 2012^{40}\end{array}$ & $\begin{array}{l}\text { Identificar fatores que influenciam encaminhamento aos serviços } \\
\text { de CP. }\end{array}$ \\
\hline $\begin{array}{l}\text { Palliative care out consultation service in a teaching hospital } \\
\text { in Bangladesh/2011 }\end{array}$ & $\begin{array}{l}\text { Determinar características de encaminhamento, padrões de } \\
\text { doença e consciência da doença e outros sintomas de pacientes } \\
\text { que frequentam serviço ambulatorial de CP. }\end{array}$ \\
\hline
\end{tabular}


maligna em pacientes com tumores ginecológicos) e também através da mensuração do número de hospitalizações e duração das internações ${ }^{33-35}$.

Ao atrelar os encaminhamentos a estas características, os pesquisadores reproduzem o modelo de encaminhamento baseado em medidas prognóstico e expectativa de vida. Embora interessantes para análise estatística, estes tendem a espelhar o modelo americano, considerado excludente e impreciso ${ }^{22}$.

Ao atribuir a um paciente expectativa de vida curta, deixamos de focar em suas reais necessidades e de sua família, correndo o risco de condená-lo a um fenômeno conhecido como "morte social". Além disso, apesar de toda tecnologia disponível ainda hoje é impossível predizer com exatidão o prognóstico de um paciente, fazendo com que haja uma tendência a superestimação. Esta visão otimista contribui, cada vez mais a favor do prolongamento na provisão dos tratamentos oncológicos, com consequente encaminhamento tardio aos $\mathrm{CP}^{22}$.

Foi raro a ocorrência de encaminhamentos a pedido de pacientes e família, demonstrando a importância da abordagem do assunto pelo profissional de saúde. Alguns serviços foram chamados de cuidados de apoio, na tentativa de superar o estigma relacionado ao nome CP. O suporte oferecido por estes serviços permitiu que pacientes tolerassem por mais tempo as terapias voltadas para a doença (como a quimioterapia) $)^{35-36}$.

Foram apontados como desafios aos modelos de encaminhamentos questões como indefinições conceituais e de dificuldade de definições e responsabilidades entre especialistas $35,37,40$, além de aspectos estruturais relacionados aos serviços (oferta heterogênea, ausência de seguro sáude e dificuldade de acesso a serviços que prestam os CP paralelo ao tratamento oncológico) ${ }^{39-41}$.

Estes assuntos são abordados no Global Atlas of Palliative Care at the End of Life elaborado pela Worldwide palliative care alliance (WPCA) e WHO em 2014 em que são apresentados aspectos relacionados a: barreiras à integração dos serviços, modelos de implementação e financiamento, além de um mapa sobre provisão e necessidades de CP mundial ${ }^{42}$.

Neste documento são citadas como barreiras para encaminhamentos: ausência de política de CP em alguns países, formação profissional deficitária, acesso inadequado ao uso de opióides e a provisão desarticulada de serviços, onde temos a associação entre provisão de CP e índice de desenvolvimento humano ${ }^{42}$.

Uma estratégia local para melhorar o acesso precoce aos CP tem sido buscada pelas entidades de classe americanas, como a American Society of Clinical Oncology, que incentiva a provisão de cuidados paliativos integrados aos cuidados oncológicos. Neste sentido estudo de $2010{ }^{43}$ produz significativa contribuição ao descrever os modelos de cuidados integrados a prática oncológica e um modelo de abordagem na comunicação de pacientes.
Foram descritos 3 modelos de cuidados: o modelo de cuidados isolado, útil em consultórios e comunidades pequenas em que o oncologista assume o cuidado ao câncer e as medidas de cuidados paliativos, porém possui a desvantagem da falta de treinamento do profissional oncologista em CP, restrições de tempo e risco de burnout do oncologista; o modelo congresso e o modelo de cuidados integrados.

No modelo congresso o oncologista faz diversos encaminhamentos a especialistas o paciente é atendido pela equipe de CP somente em fase de fim de vida. Esse modelo possui as desvantagens de ser cansativo e dispendioso para pacientes e familiares e, pela falta de interação entre especialistas pode resultar em prescrições conflitantes, interações medicamentosas e agravamento ao invés de resolução de problemas. No modelo de cuidados integrados temos o oncologista atuando em questões relacionadas ao câncer e o paliativista atuando na vasta maioria das questões psicológicas e físicas. Essa abordagem traz mais benefícios a oncologistas, pacientes e familiares e apresenta de uma forma prática a viabilidade do tema desta revisão ${ }^{43}$.

Outra contribuição apresentada neste $\operatorname{artigo~}^{43}$ foi a abordagem de pacientes sobre prognóstico e metas do cuidado. Esta abordagem faz analogia a compra de um carro em que devem ser abordados questões sobre imprevistos e medidas de conforto. Embora bastante criativo, não é possível conhecer sua aplicabilidade a prática oncológica brasileira uma vez que não se tem conhecimento de seu uso na nossa literatura.

Sendo assim, foram considerados como facilitadores dos encaminhamentos aos CP: pacientes com altas cargas de sintomas e pior prognóstico, e aspectos relacionados a prática oncológica como: experiências prévias positivas de encaminhamento, visão de que médicos paliativistas dispõem de mais tempo para acessar questões complexas. A mudança no nome do serviço para serviço de suporte, ao invés de cuidados paliativos, também trouxe aumento nos encaminhamentos.

Foram consideradas barreiras para encaminhamentos: concepção de que os CP constituem uma filosofia alternativa, incompatível com a terapia para o câncer, crença de que a responsabilidade pela provisão dos CP é do oncologista e falta de conhecimentos sobre serviços de CP disponíveis e a indisponibilidade de acesso a serviços que prestem atendimento integrado (paralelo ao tratamento oncológico padrão).

Na prática ficou demonstrado que a visão da integração entre cuidado paliativo e cuidado oncológico padrão ainda não é uma unanimidade. No Brasil, principalmente com relação ao modelo de provisão de $\mathrm{CP}$ que é considerado de provisão isolada e desarticulada ${ }^{42}$, e devido a própria história recente da sua criação (a partir de 1980)²3, será necessário não só lutar contra barreiras, mas talvez partir da construção de uma cultura paliativa que seja adaptada às nossas necessidades e à realidade dos serviços de saúde. 


\section{CONCLUSÕES}

O debate sobre o momento ideal para encaminhamento aos cuidados paliativos cresceu como reflexo do surgimento das novas tecnologias para prolongamento de sobrevida e apoia-se no ideal do encaminhamento precoce. Este foi associado a diversos benefícios, porém sua prática parece ainda em evolução. Mesmo em locais que dispõem de serviços de cuidados paliativos integrados aos cuidados oncológicos, ainda vivenciamos os encaminhamentos tardios. A população de pacientes com tumores hematológicos encontra-se mais vulnerável a esse tipo de encaminhamento.

Existem elementos facilitadores aos encaminhamentos, relacionados ao alívio de sintomas e a experiência prévia positiva com estes. Porém como barreiras surgiram a falta de oferta e aspectos conceituais que necessitaram ser abordados na tentativa de expandir este cuidado a todos os pacientes com câncer que os necessitam.

Serão necessárias várias ações de âmbito global para aproximar o paciente sem possibilidade de cura do cuidado paliativo, como mudanças políticas que favoreçam encaminhamentos precoces e aumentem a oferta de cuidados paliativos integrados. Paralelamente, deve-se implementar ações locais com o objetivo de conscientização dos profissionais de saúde da importância dos cuidados paliativos e articulação entre o papel do oncologista e do especialista na realização destes cuidados.

\section{REFERÊNCIAS}

1. Ministério da Saúde (BR), Instituto Nacional de Câncer José Alencar Gomes da Silva - INCA; Coordenação de Prevenção e Vigilância. Rio de Janeiro. Estimativa 2014: incidência de câncer no Brasil. Rio de Janeiro, RJ: INCA; 2013. Disponível em: http://www.saude.sp.gov.br/resources/ses/perfil/gestor/ homepage/outros-destaques/estimativa-de-incidencia-decancer-2014/estimativa_cancer_24042014.pdf.

2. Polovich M, Olsen M, LeFebre KB. Chemotherapy and Biotherapy Guidelines and recommendations for practice. 4th ed. Pittsburgh: Oncology Nursing Society, 2014.

3. Bonassa EMA,Gato,MIR. Terapêutica oncológica para enfermeiros e farmacêuticos. $4^{\mathrm{a}}$ ed. Rio de Janeiro: Atheneu; 2012.

4. Souza, RS. Pacientes oncológicos em quimioterapia paliativa: perfil e relações entre sintomas, capacidade funcional e qualidade de vida. [dissertação mestrado]. Belo Horizonte: Universidade Federal de Minas Gerais, Escola de Enfermagem, 2011. [Internet] Disponível em: http://www.bibliotecadigital. ufmg.br/dspace/bitstream/handle/1843/GCPA-8NGJEU/ raissa_silva_souza.pdf?sequence $=1$.

5. World Health Organization. Who definition of palliative care. WHO [Internet] [cited 2014 feb 10]. Available from: http:// www.who.int/cancer/palliative/definition/en/.

6. Ferrell BR, Coyle N. Oxford Textbook of palliative nursing. 3rd ed. USA: Oxford University Press; 2010.

7. Peppercorn JM, Smith TJ, Helft PR, DeBono DJ, Berry SR, Wollins DS et al. American Society of Clinical Oncology Statement:
Toward Individualized Care for Patients With Advanced Cancer. J Clin Oncol 2011, 29 (6): p. 755-760.

8. Santos CMC, Pimenta CAM, Nobre MRC. A estratégia PICO para a construção da pergunta de pesquisa e busca de evidências. Rev. Latino-Am Enfermagem ;15(3), São Paulo, Jun, 2007. Disponível em: http://www.scielo.br/pdf/rlae/v15n3/ pt_v15n3a23.pdf.

9. Christovam Barbara Pompeu, Porto Isaura Setenta, Oliveira Denise Cristina de. Gerência do cuidado de enfermagem em cenários hospitalares: a construção de um conceito. Rev. esc. enferm. USP [Internet]. 2012 June [cited 2016 June 11]; 46(3): 734-741. Available from: http://www. scielo.br/scielo.php?script=sci_arttext \&pid=S008062342012000300028\&lng=en. http://dx.doi.org/10.1590/ S0080-62342012000300028.

10. Mendes Karina Dal Sasso, Silveira Renata Cristina de Campos Pereira, Galvão Cristina Maria. Revisão integrativa: método de pesquisa para a incorporação de evidências na saúde e na enfermagem. Texto contexto - enferm. [Internet]. 2008 Dec [cited 2016 June 11] ; 17( 4): 758-764. Available from: http:// www.scielo.br/scielo.php?script=sci_arttext \& pid=S010407072008000400018\&lng=en. http://dx.doi.org/10.1590/ S0104-07072008000400018.

11. Kistler EA, Morrison RS, Richardson LD, Ortiz JM, Grudzen CR. Emergency department-triggered palliative care in advanced cancer: proof of concept. Acad emerg. med. [Internet] 2015 feb; 22(2):237-9. In: PubMed; PMID: 25639187.

12. Rugno FC, Paiva BS, Paiva CE. Early integration of palliative care facilitates the discontinuation of anticancer treatment in women with advanced breast or gynecologic cancers. Gynecol oncol. [Internet] 2014 nov; 135(2):249-53. Available from: https://www.sgo.org/wp-content/uploads/2012/09/Rugnoet-al-Early-integration.pdf.

13. Hui D, Kim SH, Roquemore J, Dev R, Chisholm G, Bruera E. Impact of timing and setting of palliative care referral on quality of end-of-life care in cancer patients. Cancer [Internet] 2014 jun; 120(11):1743-9. Available from: http://onlinelibrary. wiley.com/doi/10.1002/cncr.28628/pdf.

14. Corbett CL, Johnstone M, Trauer JM, Spruyt O. Palliative care and hematological malignancies: increased referrals at a comprehensive cancer centre. J Palliat Med. [Internet] 2013 may; 16(5):537-41. In: PubMed; PMID: 23822210.

15. Otsuka M,Koyama A,Matsuoka H,Niki M,Makimura C,Sakamoto R, Sakai K, Fukuoka M. Early palliative intervention for patients with advanced cancer. Jpn. J. Clin. Oncol. [Internet] 2013 aug; 43(8):788-94. Available from: http://jjco.oxfordjournals.org/ content/43/8/788.full.pdf+html.

16. Reyes-Gibby CC1, Anderson KO, Shete S, Bruera E, Yennurajalingam S. Early referral to supportive care specialists for symptom burden in lung cancer patients: a comparison of non-Hispanic whites, Hispanics, and non-Hispanic blacks. Cancer. [Inernet] 2012 feb 1; 118(3):856-63. Available from: http://onlinelibrary.wiley.com/doi/10.1002/cncr.26312/pdf.

17. Bell CL, Kuriya M, Fischberg D. Hospice referrals and code status: outcomes of inpatient palliative care consultation among asian americans and pacific islanders with cancer. J Pain Symptom Manage. [Internet] 2011 oct; 42(4):557-64. Available from: http://www.jpsmjournal.com/article/S08853924(11)00134-5/fulltext. 
18. Jacobsen J, Jackson V, Dahlin C, Greer J, Perez-Cruz P, Billings JA, Pirl W, Temel J. Components of early outpatient palliative care consultation in patients with metastatic nonsmall cell lung cancer.J Palliat Med. 2011 Apr;14(4):459-64. In: PubMed; PMID: 21417739.

19. Fukui S, Fujita J, Tsujimura M, Sumikawa Y, Hayashi Y, Fukui N. Late referrals to home palliative care service affecting death at home in advanced cancer patients in Japan: a nationwide survey. Ann Oncol. [Internet] 2011 sep; 22(9):2113-20. Available from: http:// annonc.oxfordjournals.org/content/22/9/2113.long.

20. Baek YJ, Shin DW, Choi JY, Kang J, Mo HN, Kim YH, Kim S, Jung KW, Joo J, Park EC. Late referral to palliative care services in Korea. J Pain Symptom Manage. [Internte] 2011 apr; 41(4):692-9. Available from: http://www.jpsmjournal.com/article/S08853924(10)01020-1/fulltext.

21. Dalal S, Palla S, Hui D, Nguyen L, Chacko R, Li Z, Fadul N, Scott C, Thornton V, Coldman B, Amin Y, Bruera E. Association between a name change from palliative to supportive care and the timing of patient referrals at a comprehensive cancer center. Oncologist [Internet] 2011; 16(1):105-11. Available from: http:// theoncologist.alphamedpress.org/content/16/1/105.long.

22. Matsumoto, DY. Cuidados Paliativos: conceitos, fundamentos e princípios In: Academia Nacional de Cuidados Paliativos. Manual de cuidados paliativos. Rio de Janeiro: Diagraphic, 2009: 14-19. Disponível em: http://www.santacasasp.org.br/ upSrv01/up_publicacoes/8011/10577_Manual\%20de\%20 Cuidados\%20Paliativos.pdf.

23. Temel JS; Greer JA; Muzikansky A; Gallagher ER; Admane S; Jackson VA; Dahlin CM; Blinderman CD; Jacobsen J; Pirl WF; Billings JA; Lynch TJ. Early palliative care for patients with metastatic non-small-cell lung cancer. $\mathrm{N}$ Eng J Med. [Internet] 2010 aug; 363(8): 733-42. Available from: http://www.nejm. org/doi/pdf/10.1056/NEJMoa1000678.

24. Araujo LHL, Baldotto CS, Zukin M, Vieira FMAC, Victorino AP, Rocha VR et al. Sobrevida e fatores prognósticos em pacientes com câncer de pulmão de células não pequenas assistidos na saúde suplementar. Rev. bras. epidemiol. [Internet] 2014; 17(4): 1001-14. Disponível em: http://www.scielosp.org/pdf/ rbepid/v17n4/pt_1415-790X-rbepid-17-04-01001.pdf.

25. Armstrong DK. Relapsed ovarian cancer: challenges and management strategies for a chronic disease. Oncologist. [Internet] 2002 oct; 7 Suppl 5:20-8. Available from: http:// theoncologist.alphamedpress.org/content/7/suppl_5/20.long.

26. Blackledge GI, Lawton F, Redman C, Kelly K. Response of patients in phase II studies of chemotherapy in ovarian cancer: implications for patient treatment and the design of phase II trials. Br J Cancer. [Internet] 1989 apr; 59(4):650-3. Available from: http://www.ncbi.nlm.nih.gov/pmc/articles/ PMC2247161/pdf/brjcancer00126-0168.pdf.

27. Cojocariu OM1, Huguet F, Lefevre M, Périé S. Facteurs pronostiques et prédictifs des cancers des voies aérogigestives supérieures. Bull Cancer. [Internet] 2009 avr; 96(4):369-78. Disponible et: http://www.jle.com/fr/revues/bdc/e-docs/ facteurs_pronostiques_et_predictifs_des_cancers_des_voies_ aerodigestives_superieures_280729/article.phtml.

28. Hung YS, Wu JH, Chang H, Wang PN, Kao CY, Wang HM et al. Characteristics of patients with hematologic malignancies who receives palliative care consultation services in a medical center. Am J Hosp Palliat Care [Internet] 2013 dec; 30(8):773-
80. In; PubMed [in process]; PMID: 23298875.

29. Ministério da Saúde (BR). Política Nacional de Atenção Oncológica. Norma Técnica revisada após CT de atenção em Saúde. [Internet] 2005 nov. Disponível em: http://bvsms. saude.gov.br/bvs/publicacoes/politica_nacional_atencao_ oncologica.pdf.

30. BRASIL. Ministério da Saúde. Portaria n 4.274/GM/MS de 30 de dezembro de 2010. Estabelece diretrizes para a Organização da rede de atenção á saúde no âmbito do Sistema Único da Saúde. Disponível em: http://bvsms.saude.gov.br/bvs/ saudelegis/gm/2010/prt4279_30_12_2010.html.

31. BRASIL. Portaria n 874/GM/MS de 16 de maio de 2013. Institui a Política Nacional para Prevenção e Controle do Câncer na rede de Atenção em Saúde das Pessoas com doenças Crônicas no âmbito do Sistema Único de Sáude. Disponível em: http://bvsms.saude.gov.br/bvs/saudelegis/ gm/2013/prt0874_16_05_2013.html.

32. O'Connor NR, Hu R, Harris PS, Ache K, Casarett DJ. Hospice admissions for cancer in the final days of life: independent predictors and implications for quality measures. J Clin Oncol [Internet] 2014 oct; 32(28):3184-9. Available from: http://jco. ascopubs.org/content/32/28/3184.long.

33. Collins A, Sundararajan V, Brand CA, Moore G, Lethborg C, Gold M, Murphy MA, Bohensky MA, Philip J. Clinical presentation and patterns of care for short-term survivors of malignant glioma. J Neurooncol [Internet] 2014 sep; 119(2):333-41. In: PubMed; PMID: 24889839.

34. Lefkowits C, Binstock AB, Courtney-Brooks M, Teuteberg WG, Leahy J, Sukumvanich P, Kelley JL. Predictors of palliative care consultation on an inpatient gynecologic oncology service: are we following ASCO recommendations? Gynecol Oncol. [Internet] 2014 may; 133(2):319-2. In: PubMed; PMID: 24594073.

35. Schenker $Y$, Crowley-Matoka M, Dohan D, Rabow MW, Smith CB, White DB, Chu E, Tiver GA, Einhorn S, Arnold RM. Oncologist factors that influence referrals to subspecialty palliative care clinics. J Oncol Pract [Internet] 2014 mar; 10(2):e37-e44. Available from: http://jop.ascopubs.org/ content/early/2013/12/02/JOP.2013.001130.full.pdf.

36. Kwon JH, Hui D, Chisholm G, Ha C, Yennurajalingam S, Kang JH, Bruera E. Clinical characteristics of cancer patients referred early to supportive and palliative care.J Palliat Med [Internet] 2013 feb; 16(2):148-55. Available from: http://www.ncbi.nlm. nih.gov/pmc/articles/PMC3569943/.

37. Peng JK, Chiu TY, Hu WY, Lin CC, Chen CY, Hung SH. What influences the willingness of community physicians to provide palliative care for patients with terminal cancer? Evidence from a nationwide survey. Jpn J Clin Oncol [Internet] 2013 mar; 43(3):278-85. Available from: http://jjco.oxfordjournals. org/content/43/3/278.long.

38. Hui D, Kim SH, Kwon JH, Tanco KC, Zhang T, Kang JH, Rhondali W, Chisholm G, Bruera E. Access to palliative care among patients treated at a comprehensive cancer center. Oncologist [Internet] 2012 dez; 17(12):1574-80. Available from: http://theoncologist. alphamedpress.org/content/17/12/1574.full.pdf+html.

39. Wentlandt K, Krzyzanowska MK, Swami N, Rodin GM, Le LW, Zimmermann C. Referral practices of oncologists to specialized palliative care. J Clin Oncol [Internet] 2012 dec; 30(35):4380-6. Available from: http://jco.ascopubs.org/ content/30/35/4380.long. 
40. Smith CB, Nelson JE, Berman AR, Powell CA, Fleischman J, Salazar-Schicchi J, Wisnivesky JP. Lung cancer physicians' referral practices for palliative care consultation. Ann Oncol [Internet] 2012 feb; 23(2):382-7. Available from: http://www. ncbi.nlm.nih.gov/pmc/articles/PMC3265546/.

41. Ahmad NU, Haque MF, Khan F, Kamal MM. Palliative care out patient consultation service in a teaching hospital in Bangladesh. Mymensingh Med J [Internet] 2011 jan; 20(1):98103. In: PubMed; PMID:21240171.

42. World Health Organization, Worldwide palliative care alliance. Global atlas of palliative care at the end of life. [Internet] 2014. [citado 2015 mar 12]. Available from: http://www.who. int/nmh/Global_Atlas_of_Palliative_Care.pdf.

43. Bruera E, Hui D. Integrating supportive and palliative care in the trajectory of cancer: establishing goals and models of care. J Clin Oncol [Internet] 2010; 28(25): 4013-17. Available from: http://jco.ascopubs.org/content/28/25/4013.short. 